\title{
Misturasotoma, a new nearctic springtail genus (Collembola: Isotomidae)
}

\author{
ERNEST C. BERNARD ${ }^{1} \&$ KENNETH A. CHRISTIANSEN ${ }^{2}$ \\ ${ }^{1}$ Entomology \& Plant Pathology, The University of Tennessee, 2431 Joe Johnson Drive, 205 Plant Sciences, Knoxville, Tennessee, \\ 37996-4560,U.S.A. E-mail: ebernard@utk.edu \\ ${ }^{2}$ Department of Biology, Grinnell College, Grinnell, Iowa, 50112-0886 U.S.A. E-mail: christak@ grinnell.edu
}

\begin{abstract}
Type specimens of Desoria brucealla (Wray), a western North American species, are transferred to Misturasotoma n.gen. The new genus differs from Desoria, Heteroisotoma, Isotoma, and Parisotoma by the following combination of characters: eyes absent, tibiotarsi with 11 setae in distal whorl, tenaculum with 9-15 setae, manubrium with apical spinelike setae, mucrones quadridentate, macrochaetae smooth, and sensilliform setae of abdominal segments IV and V short.
\end{abstract}

Key words: Isotoma brucealla, Isotoma brucei, North America, taxonomy, Utah, Washington

\section{Introduction}

In the course of revising The Collembola of North America North of the Rio Grande (CONANRG) we were unable to confidently place Isotoma brucealla Wray, 1953b in any genus of Isotomidae, based on the key of Potapov (2001). In earlier versions of CONANRG (Christiansen \& Bellinger 1981, 1998) this species had been placed in the subgenus Desoria Agassiz \& Nicolet. Type specimens were not available at the time and placement of this taxon in Desoria was based on specimens collected in a lava tube cave in Skamania County, Washington.

Wray (1953a) described Isotoma brucei from pocket gopher nests in Utah, but almost immediately realized that this name was preoccupied (Carpenter 1907). Therefore, he renamed the species I. brucealla (Wray 1953b). Recently the first author was able to borrow the type specimens of I. brucealla from the North Carolina Department of Agriculture Collection (NCDA) and examine them. The NCDA collection contained three specimens labelled "Isotoma $\mathrm{n}$. sp./type series" with the locality, habitat, and collector corresponding to the collection data in the original 1953 paper. However, the collection also contained two specimens labelled "I. brucealla," collected a few days earlier than the type series but from the same habitat and by the same collector. All of the slides are labelled in Wray's hand. These latter two specimens agree well with the lava tube specimens from Washington.

All five specimens were difficult to study due to the old, dark-brown, cracked medium in which they were mounted. Each slide was placed in a moist chamber for two days to soften the old medium, then the cover slip was carefully lifted and the specimen was removed and placed in Andre I solution for 15-30 minutes. It was remounted in fresh Hoyer's medium on the same slide with the original labels and the slide was placed in a $55^{\circ} \mathrm{C}$ oven for three days to harden the medium. The cover slip then was sealed with Glyptal.

According to Wray (1953a) this taxon lacks eyes, has a narrowly elliptical postantennal organ (PAO), possesses a prominent inner unguicular tooth, and has 10-15 setae on the tenaculum. Of the three "type series" specimens, one has 8 ocelli on each side, and none of the three possesses these differentiating characters. However, the two specimens labelled "I. brucealla" fit these characters closely, and so we consider them to be the actual type specimens. In our opinion, Wray probably confused the specimens in the labeling process, which led to an error in recording the collection data that related to the original description. Therefore, we designate a lectotype and paralectotype from the two specimens that fit Wray's description. 
The types do not appear to belong to any recognised genus. They have some characters of four different isotomid genera but differ clearly from all of them in other characters. They have the distal manubrial spines characteristic of Isotoma Bourlet and Heteroisotoma Stach, but lack eyes (8 per side are characteristic of Isotoma). Both of these last two genera have tridentate mucrones (sometimes a minute apical fourth tooth in Isotoma), whereas I. brucealla has 4 well-developed mucronal teeth. Both Isotoma and Heteroisotoma have ciliate or serrate macrochaetae on the abdomen, whereas the macrochaetae of I. brucealla are smooth. In addition, Heteroisotoma spp. have a narrow, double-walled postantennal organ (PAO) (Potapov \& Stebaeva 1991), but $I$. brucealla has a more oval PAO with weakly thickened walls. Most species of Heteroisotoma have long sensilliform setae on the fifth abdominal tergite, while I. brucealla has short sensilliform setae. Desoria has mucrones similar to $I$. brucealla, but is defined as having at least $6+6$ eyes and lacking the spinelike distal manubrial setae seen in I. brucealla. Parisotoma Bagnall, has reduced eye number but the tenaculum has 6 or fewer setae, in contrast to the 9-15 found in I. brucealla. In addition, most species of Parisotoma have tridentate mucrones, all have ciliate macrochaetae, and all lack the spine-like distal manubrial setae of I. brucealla. After long consideration we have concluded that the most parsimonious action is to create a new genus for the species.

Antennal segments are abbreviated as Ant. I-IV. Abdominal segments are abbreviated as Abd. I-VI. Labial palpus setae and papillae are labelled according to Fjellberg (1998).

\section{Misturasotoma new genus}

Diagnosis. Isotominae with all abdominal segments clearly separate (Fig. 1). Eyes absent. Postantennal organ elongate-elliptical. Antenna without apical bulb or clearly differentiated blunt setae. Tenaculum quadridentate with 9-15 setae on corpus. Unguis with prominent inner tooth and pair of external dorsal teeth. Unguiculus with prominent corner tooth. Manubrium with many setae on both dorsal and ventral surfaces; some apical setae spine-like. Dentes slender, tapering, dorsally crenulate and about twice as long as manubrium, with many ventral setae. Mucro quadridentate with all teeth well developed. All body setae smooth and acuminate; abdominal sensilliform setae short, thin.

Type species: Isotoma brucealla Wray 1953b.

Discussion. Wray (1953a) originally described this species as Isotoma brucei but upon discovery of a previously described Isotoma brucei (Carpenter, 1907), now Archisotoma brucei, changed the name to $I$. brucealla (Wray 1953b). In Potapov (2001) this genus will key to Heteroisotoma but differs in having smooth rather than ciliate setae and an oval PAO with thin walls rather than a narrow PAO with thick walls. The arrangement of the abdominal sensilla also seems to differ from Heteroisotoma in that the type species has anterior sensilliform setae on Abd. IV and 3+3 anterior sensilliform setae on Abd. V; Heteroisotoma spp. lack anterior sensilliform setae on Abd. IV and have 2+2 anterior sensilliform setae on Abd. V.

Etymology. From the Latin mistura $=$ mixture and Isotoma, referring to the mixture of characteristics of different genera of Isotominae found in the genus.

\section{Misturasotoma brucealla (Wray 1953a)}

Figs. 1-21

Isotoma brucei Wray, 1953a: 66 (nec Carpenter 1907: 474).

Isotoma brucealla Wray, 1953b: 55.

Isotoma (Desoria) brucealla Christiansen \& Bellinger, 1981: 718; 1998: 797.

Parisotoma brucealla Bellinger et al., 1996-2010: www.collembola.org.

Material examined. Lectotype, USA, Utah, Monte Cristo, 15 July 1951, pocket gopher nest, G. F. Knowlton and T. T. Tibbetts, colls. Paralectotype, same data except collected 18 July 1951. Both specimens are deposited in the North Carolina State University Entomology Collection, Raleigh, North Carolina, USA. One 
specimen collected from USA, Washington, Skamania County, lava tube cave, is deposited in the Grinnell College, Grinnell, Iowa, Collembola collection.

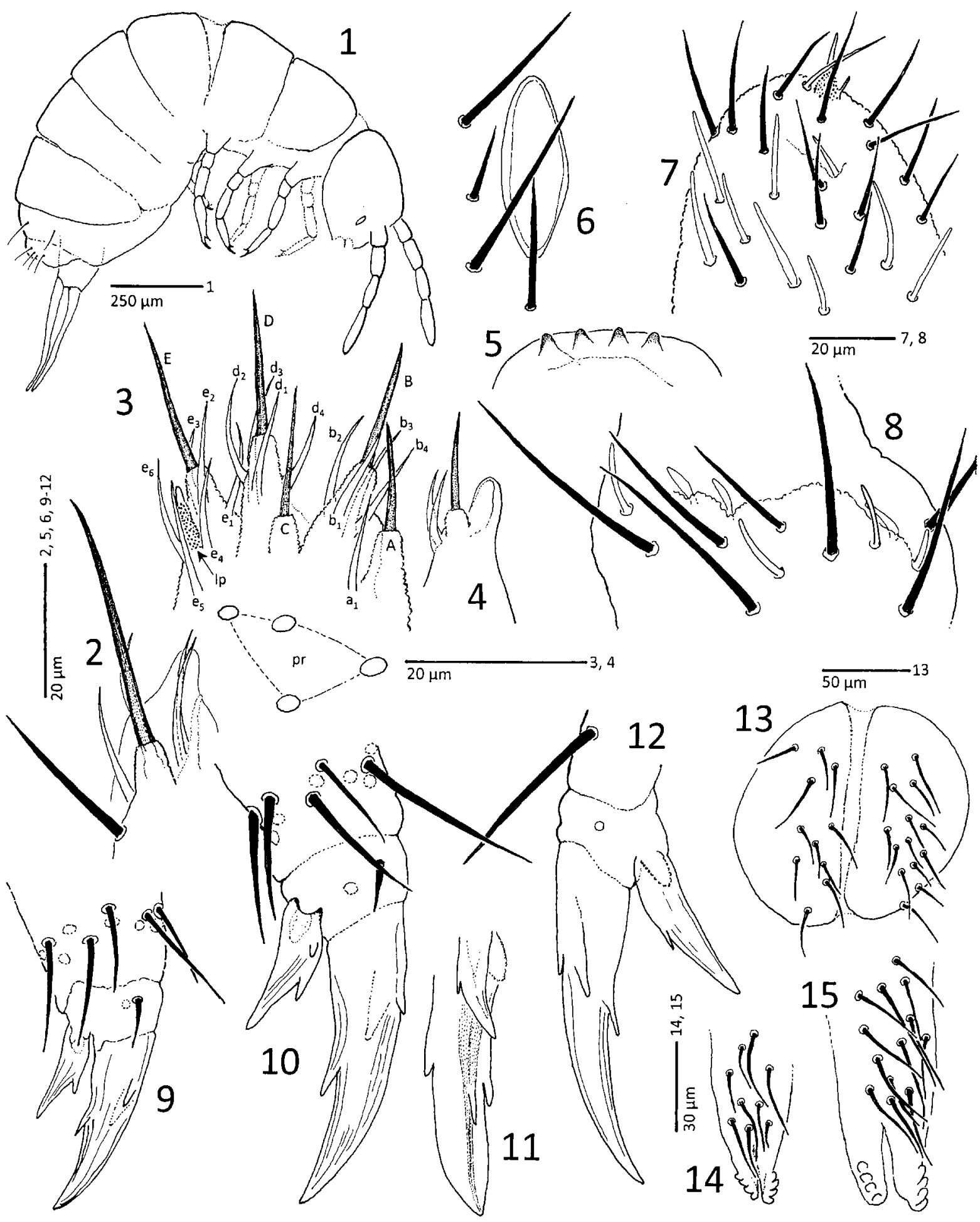

FIGURES 1-15. Misturasotoma brucealla. 1. Habitus, lectotype. 2. Maxillary palpus, paralectotype. 3. Labial palpus, paralectotype. 4. Hypostomal lobe, paralectotype. 5. Apex of labrum, paralectotype. 6. Postantennal organ, lectotype. 7. Apex of fourth antennal segment, lectotype. 8. Sense organ of third antennal segment, lectotype. 9. Forefoot complex, lectotype. 10. Hind foot complex, paralectotype. 11. Hind foot, ventral view, paralectotype. 12. Hind foot complex, Washington specimen. 13. Ventral tube, ventral view, paralectotype. 14. Tenaculum, lectotype. 15. Tenaculum, paralectotype. 


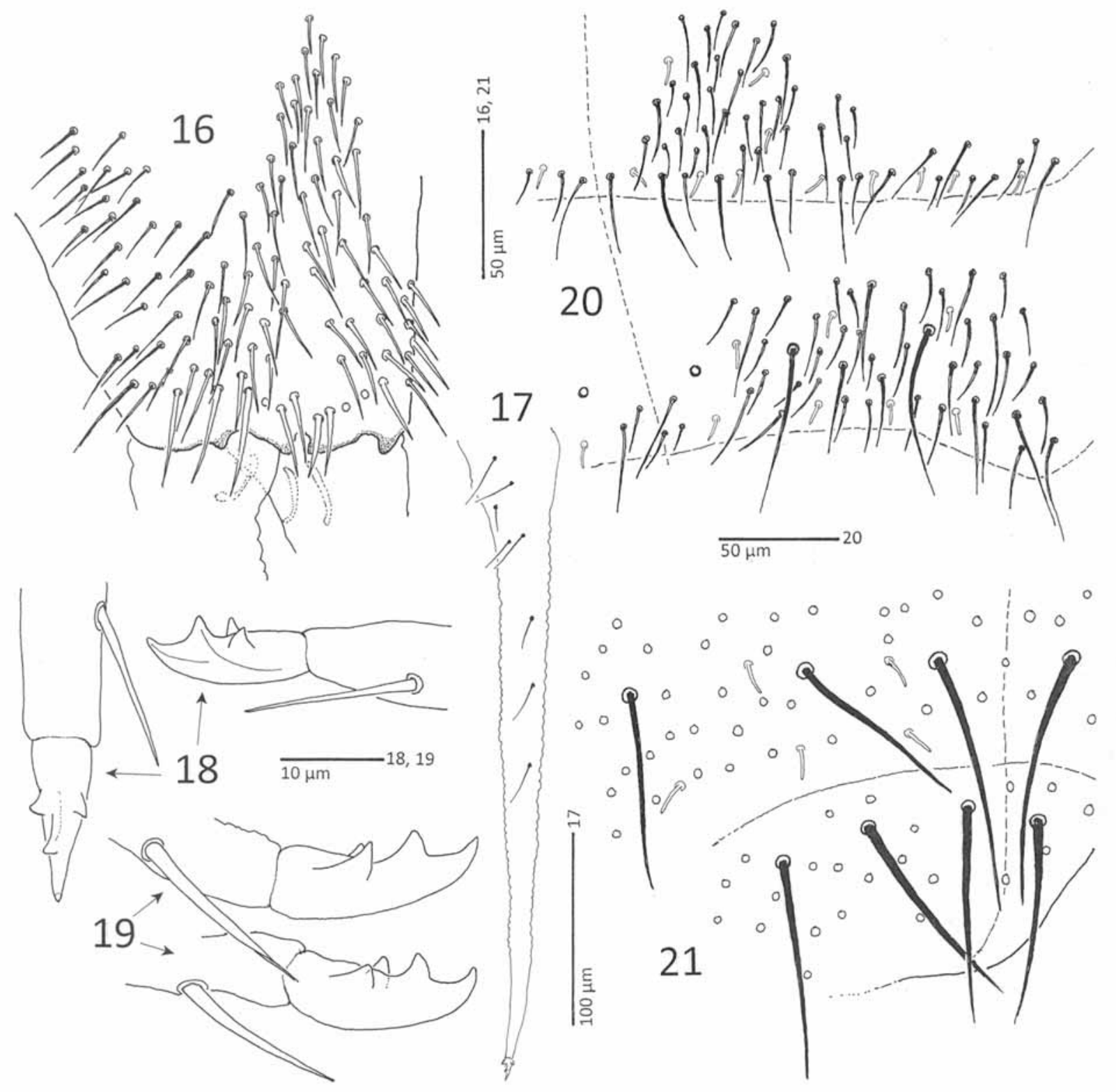

FIGURES 16-21. Misturasotoma brucealla. 16. Manubrium, ventral view, paralectotype. 17. Dens, dorsal view, lectotype. 18. Mucrones, lectotype. 19, Mucrones, paralectotype. 20. Chaetotaxy of Abd. IV and Abd. V tergites, right side, lectotype; typical setae not near sensilliform setae omitted. 21. Chaetotaxy of Abd. V and Abd. VI tergites, left side, paralectotype, only macrochaetae and sensilliform setae drawn.

Redescription. Colour: "Yellowish-white...with traces of black pigment over head and body in the pattern of small grouped areas of punctiform pigment..." (Wray 1953a). Labrum with 5-5-4 setal arrangement; apex of labrum rounded-trapezoidal with four subapical tubercles and a slightly more proximal transverse ridge (Fig. 5). All maxillary lamellae short, none exceeding tips of capitular teeth. Labial palpus (Fig. 3) with 4 proximal setae and all guard setae except $\mathrm{e}_{7}$; lateral papilla minutely granulated on basal twothirds, smooth apically. Hypostomal papilla (Fig. 4) with apical seta and two proximal unsocketed setae. Maxillary palpus (Fig. 2) with long apical seta, four unsocketed setae, and one socketed basal seta. First antennal segment ventrally with loose cluster of 6-8 pointed setulae. Sense organ of Ant. III with two slender, 
weakly bent or straight sense clubs in shallow groove, two similar sensilla subventrally, and three longer, slightly more proximal sensilla (Fig. 8). Fourth antennal segment with numerous slender sensilliform setae not sharply distinguished from typical setae; subapical sense rod long, slender, pointed; pin seta broadly conical basally, slender distally, with small linear secondary seta near base (Fig. 9). P.A.O. elongate-elliptical, with 4 guard setae (Fig. 6). Eyes absent. Length of lectotype $1.6 \mathrm{~mm}$, paralectotype $2.1 \mathrm{~mm}$.

Each tibiotarsus with 11 apical setae, tenent hair acuminate (Figs. 9, 10, 12). Unguis with strong ventral tooth and two subdorsal teeth not forming a tunica, one longer and stronger than the other; unguiculus with strong inner tooth and on some legs with small basal tooth; unguicular tip narrowly rounded (Figs. 9, 10). Unguis broad in ventral view (Fig. 11). Abdomen with third and fourth segments about equal in length; fifth and sixth segments clearly demarcated. Ventral tube with 24 (10+14) setae (Fig. 13). Tenaculum with 9-13 setae (Figs. 14, 15). Ventro-apical manubrial setae spinelike, slightly thicker than other manubrial setae (Fig. 16). Dens about 2.5 times longer than manubrium, with 8 dorsal setae proximally and in mid-region, without seta in distal region (Fig. 17); ventro-apical seta reaching to level of mucronal basal teeth. Mucro quadridentate, apical and subapical teeth subequal in size; basal teeth smaller, lateral, not aligned with subapical tooth (Figs. 18, 19).

All setae smooth. Macrochaetae distinctly developed only on last three abdominal terga, each with $3+3$ macrochaetae (Figs. 20,21). Macrochaetae of last two abdominal segments about 2.5 times length of unguis. Sensilliform setae of Abd. IV and V of lectotype distributed as follows: Abd. IV, $2+2$ anterior, $0+1$ medial, $8+8$ posterior; Abd. V, $2+3$ anterior, $3+4$ posterior (Fig. 20). On Abd. V of paralectotype, $2+2$ anterior, $3+3$ posterior sensilliform setae (Fig. 21). Arrangement and number of sensilliform setae on other segments not determined.

Remarks. The extremely flattened, partially twisted condition of the type specimens did not allow for an accurate determination of sensilliform seta distribution on segments anterior to Abd. IV. Sex of the types is unknown, as the genital aperture of each specimen was obscured by the overlapping abdominal terminus and extended furcula. Likewise, several features, e.g., the ventral tube chaetotaxy and the mouthparts, could only be determined from one of the specimens. The lectotype was chosen for the clarity of the abdominal chaetotaxy and antennal structures, even though it is significantly shorter than the paratype and possibly is an immature specimen.

The exact type locality of this species is unknown. In Utah, "Monte Cristo" currently refers to a large recreational area and snowmobile trail north of Huntsville, Utah, in the Uinta-Wasatch-Cache National Forest. It is also the name of a mountain range in the same area.

Christiansen \& Bellinger $(1981,1998)$ based their understanding of M. brucealla on a specimen collected from a lava tube in Washington, as the type specimens were unavailable at the time. This specimen was reexamined and found to be very similar to the M. brucealla types except that the ungues are more slender. The arrangement of the visible sensilliform setae of Abd. IV and V appeared to be compatible with that of the types but could not be determined in its entirety. This specimen should still be considered to be M. brucealla.

\section{References}

Bellinger, P. F., Christiansen, K. A. \& Janssens, F. (1996-2010) Checklist of the Collembola of the world. Available from http://www.collembola.org/taxa/collembo.htm (accessed 10 February 2010).

Carpenter, G. H. (1907) Collembola from the South Orkney Islands. Proceedings of the Royal Society of Edinburgh, 26, 473-483.

Christiansen, K. \& Bellinger, P. (1981) The Collembola of North America north of the Rio Grande. Grinnell College, Grinnell, Iowa, $1322 \mathrm{pp}$.

Christiansen, K. \& Bellinger, P. (1998) The Collembola of North America north of the Rio Grande, 2nd edition. Grinnell College, Grinnell, Iowa, $1520 \mathrm{pp}$.

Fjellberg, A. (1998) The labial palp in Collembola. Zoologischer Anzeiger, 237, 309-330.

Potapov, M. (2001) Synopses on Palaearctic Collembola Vol. 3 - Isotomidae. Abhandlungen Berichten Naturkundemuseum, Görlitz, 73, 1-603. 
Potapov, M. \& Stebaeva, S. (1991) Redescription and systematic position of Heteroisotoma Stach (Collembola: Isotomidae). Entomologica Scandinavica, 22, 427-431.

Wray, D. L. (1953a) Two new North American Isotoma (Collembola) and key to eyeless forms. Bulletin of the Brooklyn Entomological Society, 48, 54-56.

Wray, D. L. (1953b) Change of specific name of Isotoma. Bulletin of the Brooklyn Entomological Society, 48, 66. 